\title{
Posttraumatic Stress and Posttraumatic Growth Among Low-Income Mothers Who Survived Hurricane Katrina
}

\author{
Sarah R. Lowe, Emily E. Manove, and Jean E. Rhodes \\ University of Massachusetts Boston
}

\begin{abstract}
Objective: The purpose of the study was to explore the relationship between posttraumatic stress (PTS) and posttraumatic growth (PTG) after Hurricane Katrina, and the role of demographics, predisaster psychological distress, hurricane-related stressors, and psychological resources (optimism and purpose) in predicting each. Method: Participants were 334 low-income mothers (82.0\% non-Hispanic Black) living in the New Orleans area prior to Hurricane Katrina, who completed surveys in the year prior to the hurricane (T1 [Time 1]) and 1 and 3 years thereafter (T2 and T3). Results: Higher T2 and T3 PTS full-scale and symptom cluster subscales (Intrusion, Avoidance, and Hyperarousal) were significantly associated with higher T3 PTG, and participants who surpassed the clinical cutoff for probable posttraumatic stress disorder at both T2 and T3 had significantly higher PTG than those who never surpassed the clinical cutoff. Older and non-Hispanic Black participants, as well as those who experienced a greater number of hurricane-related stressors and bereavement, reported significantly greater T3 PTS and PTG. Participants with lower T2 optimism reported significantly greater T3 intrusive symptoms, whereas those with higher T1 and T2 purpose reported significantly greater T3 PTG. Conclusions: Based on the results, we suggest practices and policies with which to identify disaster survivors at greater risk for PTS, as well as longitudinal investigations of reciprocal and mediational relationships between psychological resources, PTS, and PTG.
\end{abstract}

Keywords: posttraumatic growth, posttraumatic stress, natural disasters, optimism, sense of purpose

Hurricane Katrina was one of the most destructive natural disasters in U.S. history, resulting in almost 2,000 deaths and over 650,000 persons displaced (Knabb, Rhome, \& Brown, 2006; U.S. Department of Commerce, 2006). The hurricane had particularly devastating effects on those living in low-income communities, including a large proportion of non-Hispanic Black residents (Elliott \& Pais, 2006; Logan, 2006). Yet, even among vulnerable groups, there was considerable variation in survivors' responses, ranging from severe posttraumatic stress (PTS) to relatively mild responses (e.g., Galea, Tracy, Norris, \& Coffey, 2008).

In light of the variation in psychological responses to disasters, researchers have broadened their focus to include a range of adaptive responses (e.g., Bonanno, 2004). Within this context, some have investigated the phenomenon of posttraumatic growth (PTG), or self-reported positive psychological changes induced by the experience and processing of a traumatic event and its after-

This article was published Online First June 10, 2013.

Sarah R. Lowe, Emily E. Manove, and Jean E. Rhodes, Department of Psychology, University of Massachusetts Boston.

The study was funded by National Institutes of Health Grants R01HD046162 and T32MH013043, and by the National Science Foundation, the MacArthur Foundation, and the Center for Economic Policy Studies at Princeton University. We thank Thomas Brock, MDRC, Christina Paxson, Elizabeth Fussell, and Mary Waters.

Correspondence concerning this article should be addressed to Sarah R. Lowe, who is now at the Department of Epidemiology, Mailman School of Public Health, Columbia University, 722 West 168th Street 720F, New York, NY 10032. E-mail: srl2143@columbia.edu math (Tedeschi \& Calhoun, 1995). These include improved interpersonal relationships, a greater sense of new possibilities, increased personal strength, heightened spirituality, and an enhanced appreciation for life. In the current study, we focused on PTS and PTG in a sample of low-income mothers, the majority of whom were non-Hispanic Black, who survived Hurricane Katrina. Drawing on a data set that includes one predisaster and two postdisaster waves, our primary aim was to explore the relationship between PTS and PTG. In light of our interest in in a full range of postdisaster psychological responses, we drew primarily on continuous measures of these constructs. However, we also included diagnostic categories of posttraumatic stress disorder (PTSD) and classified participants into low, moderate, and severe PTS to assess differences in PTG among these groups. The secondary aim of the study was to explore the role of demographics, predisaster psychological distress, hurricane-related stressors, and psychological resources in predicting both PTS and PTG.

\section{The PTG-PTS Relationship}

In their model of PTG, Tedeschi and Calhoun (1995) proposed that when people experience a trauma severe enough that it takes a central place in their lives; changes their worldviews, assumptions, and schemas; and shifts their self-identities, the consequent distress they experience may provoke cognitive processing that results in personal growth. The model assumes that in order for PTG to manifest, some degree of psychological distress and disruption, indicative of PTS, is necessary. It is theorized that in the initial aftermath of the trauma, the overall degree of PTS must be severe enough to challenge survivors' core beliefs, which would then prompt both intrusive and deliberate cognitive processing 
(Lindstrom, Cann, Calhoun, \& Tedeschi, 2013). Thus, over time, the intrusion symptom cluster of PTS - which has been used as a measure of intrusive cognitive processing - might be the most strongly associated with PTG of the three PTS clusters (Shigemoto \& Poyrazli, in press). To date, studies exploring the relationship between the intrusion subscale of posttraumatic stress inventories and PTG have produced mixed findings, with some detecting significant positive associations with PTG (e.g., Taku, Cann, Tedeschi, \& Calhoun, 2009; Tomich \& Helgeson, 2012) and others nonsignificant or even negative associations (e.g., Dekel, Ein-Dor, \& Solomon, 2012; Stockton, Hunt, \& Joseph, 2011).

A potential explanation for these divergent findings is that, as some have argued (e.g., Hobfoll, Hall, et al., 2007; Zoellner \& Maercker, 2006), PTG might in some cases represent functional growth, associated with constructive, adaptive, and meaningful changes in survivors' lives, whereas in others it might represent illusory growth, associated with self-deception, positive illusions, and avoidance. From this standpoint, positive associations between PTS intrusion and PTG would reflect the cognitive processing indicative of functional growth, and positive associations between PTG and the other components of PTS (avoidance and hyperarousal) would reflect avoidant coping indicative of illusory growth (Dekel et al., 2012). Some studies have indeed shown that higher avoidance and hyperarousal symptoms were associated with higher PTG (Dekel et al., 2012; Wang, Wang, Wang, Wu, \& Liu, 2013). Moreover, according to the Janus Face model of self-perceived growth (Maercker \& Zoellner, 2004), the functional and illusory sides of PTG are not mutually exclusive but, rather, can co-occur, a contention that is consistent with studies finding positive associations between PTG and all three of the PTS symptom clusters (e.g., Xu \& Liao, 2011).

More generally, research literature linking overall PTS and PTG has produced mixed findings, with some studies showing positive associations (e.g., Solomon \& Dekel, 2007), others negative associations (e.g., Kimhi, Eshel, Zysberg, \& Hantman, 2010), and others curvilinear relationships, with the highest levels of PTG appearing at moderate levels of PTS (e.g., Butler et al., 2005). These inconsistencies in associations between PTG and PTS total and subscales could be due to the cross-sectional nature of most disaster studies (Bostock, Sheikh, \& Barton, 2009). To date, few studies have investigated relationships between PTS and PTG using a longitudinal design. One exception is a study by Dekel et al. (2012), who found support for a model in which initial levels and increases in PTS predicted increases in PTG and in which initial levels of hyperarousal predicted increases in PTG, whereas this was not the case for intrusion or avoidance.

\section{Predictors of PTG and PTS}

Variations in demographic and psychological resources might also render some individuals less likely to experience PTS and more likely to experience PTG. For example, associations between PTS, PTG, and demographic variables, such as ethnic minority status, younger age, and number of children in a survivors' custody, have been detected (e.g., Brewin, Andrews, \& Valentine, 2000; Xu \& Liao, 2011), although the findings regarding demographic variation in PTG have been notably inconsistent (Linley \& Joseph, 2004). More consistently, researchers have found that higher levels of exposure to traumatic events and trauma-related stressors predict both PTG and PTS (Galea et al., 2008; Xu \& Liao, 2011). In the case of natural disasters, survivors who have experienced the loss of a loved one have also been shown to be more likely to exhibit PTG and PTS (Xu \& Liao, 2011).

Psychological resources, such as optimism and sense of purpose, are also thought to differentially affect survivors' responses. Whereas optimism is negatively associated with experiences of PTS (e.g., Ahmad et al., 2010), it has been found to be positively associated with PTG (e.g., Prati \& Pietranoni, 2009). Optimism is thought to facilitate positive reappraisal of trauma, adaptive coping, and greater perceptions of social support, all of which could protect against adverse psychological responses and promote subjective experiences of PTG and reengagement in meaningful life goals (Prati \& Pietranoni, 2009). In a similar vein, survivors who are oriented toward future goals and who approach their lives with a sense of purpose might be most able to grow from their experiences. Like optimism, a sense of purpose is likely to facilitate adaptive coping, one of the most consistent predictors of PTG (Linley \& Joseph, 2004). A sense of purpose might also lead survivors to engage in volunteerism or other value-based action, which may contribute to greater posttraumatic adjustment and growth (Hobfoll et al., 2007). Indeed, Hobfoll et al. (2007) have argued that value-based action might be the key in differentiating survivors who experience functional PTG and illusory PTG. In their study of survivors of terrorist attacks and forced evacuation, those who took part in resistance efforts, "dedicating their lives through acting fully and with greater sacrifice on their beliefs" ( $p$. 356), and who manifested PTG as a result, had significantly decreased odds of developing PTSD. Conversely, a lack of purpose seems to foster lower confidence in one's ability to cope in the aftermath of trauma (Linley \& Joseph, 2011) and has been associated with higher PTS symptoms (Owens, Steger, Whitesell, \& Herrera, 2009).

It is important to note that these psychological resources are not static entities and may change in the aftermath of disasters and other traumatic events (e.g., Burger \& Palmer, 1992). Studies on the shared and unique predictors of PTG and PTS to date have been limited by a lack of predisaster data, and therefore it is unknown whether initial levels of psychological resources, or preto postdisaster changes in resources, lead to PTS and PTG. In a similar vein, studies have not simultaneously explored the role of predisaster psychological functioning in predicting PTS and PTG. This is a significant limitation given that studies including predisaster data have shown consistent associations between pre- and postdisaster psychological functioning, such that survivors with preexisting symptoms are at a heightened vulnerability to postdisaster symptoms (e.g., Weems et al., 2007). To our knowledge, no studies have explored the role of predisaster variables, including predisaster psychological symptoms and psychological resources, in predicting PTG.

\section{Current Study}

The primary purpose of this study was to investigate the relationship between PTS (both total PTS and PTS symptom clusters) and PTG, concurrently and prospectively. PTS was measured 1 and 3 years after Hurricane Katrina, and PTG was measured 3 years post-Katrina. We examined linear and curvilinear associations between PTG and PTS and made no a priori hypotheses, 
given the inconsistent findings in the literature to date. Additionally, we explored how patterns of PTS from 1 to 3 years postdisaster related to PTG and, given the lack of research on this topic, made no specific hypotheses.

A secondary aim was to explore demographic variables, hurricane-related stressors, and psychological resources as predictors of PTS and PTG. We expected that demographic and disasterrelated factors would predict both constructs in the same direction, with ethnic minority status, younger age, greater number of children, and higher levels of disaster exposure associated with higher PTS and PTG. A strength of the study was that we included predisaster assessments of psychological distress and psychological resources (optimism and purpose). We expected that higher predisaster psychological distress would significantly predict higher PTS, whereas we made no hypothesis regarding predisaster distress and PTG due to aforementioned limitations of prior research. Given that psychological resources could enable participants to adaptively cope with hurricane-related stressors, we expected survivors with higher levels of predisaster optimism and purpose to have higher PTG but lower PTS. We also expected participants who maintained higher levels of optimism and purpose to have higher PTG and lower PTS.

\section{Method}

\section{Participants and Procedure}

Institutional review boards from MDRC, Harvard University, Princeton University, and University of Massachusetts Boston approved the study. Participants were initially part of a study of low-income parents who had enrolled in three community colleges in the city of New Orleans in 2004-2005. The purpose of this initial study was to examine whether modest performance-based scholarships affected the academic achievement, health, and wellbeing of these parents (Richburg Hayes et al., 2009). To be eligible for the study, students had to be between the ages of 18 and 34; be parents of at least one dependent child under 19; have a household income under $200 \%$ of the federal poverty level; and have a high school diploma or equivalent. Students were recruited through a general marketing and outreach campaign, which included flyers, newspaper and radio announcements, and oral presentations in mandatory orientation and testing sessions for incoming first-year students. Although the program eligibility verification process, which included proof of income and parental status, may have deterred some students from enrolling, no students refused random assignment after eligibility had been determined (Richburg Hayes et al., 2009). At baseline (i.e., on enrollment in the study and prior to random assignment) participants provided primarily demographic information (e.g., age, race, number of children).

By the time Hurricanes Katrina and Rita made landfall on August 29, 2005, and September 24, 2005, respectively, 492 participants had been enrolled in the program long enough to complete a 12-month, predisaster follow-up survey (Time 1, or T1). Trained interviewers conducted the survey, which included measures of psychological distress, optimism, and purpose, over the phone and compensated participants with $\$ 20$ gift cards. After Hurricanes Katrina and Rita, between May 2006 and March 2007, 402 of these 492 participants $(81.7 \%)$ were successfully located and surveyed. Trained interviewers administered the postdisaster survey (Time 2, or T2), which included the same questions as the 12-month follow-up survey, as well as a module of hurricanerelated stressors and a measure of PTS, and sent participants \$50 gift cards. Approximately 3 years after the hurricanes, between April 2009 and March 2010, trained researchers administered an additional follow-up survey over the phone and compensated participants with $\$ 50$ gift cards for their participation (Time 3, or T3). The T3 survey included measures of PTS and PTG, and 409 of the 492 participants $(83.1 \%$ ) from T1 completed the survey. All participants provided written consent to be part of the original study, and verbal consent to participate in the postdisaster surveys. In the current study, only participants who completed all three surveys were included ( $n=348 ; 70.7 \%$ of the T1 sample). Of these participants, the subsample of male participants $(n=14$, $4.0 \%$ ) was dropped in light of consistent findings of gender differences in psychological distress following natural disasters (e.g., Norris et al., 2002).

The mean age of the final sample of 334 women at baseline was $25.54(S D=4.45)$ and their average number of children at the -year1 follow-up was $1.96(S D=1.16)$. All of the participants were living in areas affected by Hurricane Katrina, and nearly half $(47.6 \%)$ reported living in areas affected by Hurricane Rita when it struck less than a month later. Most participants (82.0\%) selfidentified as non-Hispanic Black, $10.2 \%$ as non-Hispanic White, and $3.5 \%$ as Hispanic.

\section{Measures}

Posttraumatic growth. PTG was measured at T3 using the 21-item Posttraumatic Growth Inventory (PTGI; Tedeschi \& Calhoun, 1996), which has been used in previous studies of disaster survivors (e.g., Tang, 2007). Participants rated the extent to which they experienced various changes as a result of Hurricane Katrina on a 5-point scale, ranging from 1 (not at all) to 5 (extremely). Cronbach's alpha reliability for the PTGI in this study was .93 at $\mathrm{T} 3$.

Posttraumatic stress. The Impact of Event Scale-Revised (IES-R), a 22-item self-report inventory of symptoms of PTSD (Weiss \& Marmar, 1997) with good psychometric properties (e.g., Creamer, Bell, \& Failla, 2003), was administered at T2 and T3 to measure PTS symptoms as a result of hurricane experiences. Scores for this scale range from 0 to 88 , with scores above 33 classified as indicating probable PTSD (Weiss \& Marmar, 1997). Participants were asked how much, over the prior week, they were distressed or bothered by experiences related to Hurricane Katrina, from 0 (not at all) to 4 (extremely). The IES-R also includes three subscales: (a) Intrusion (eight items, e.g., "Pictures about it popped into my mind"), (b) Avoidance (eight items, e.g., "I stayed away from reminders about it"), and (c) Hyperarousal (six items, e.g., "I was jumpy and easily startled"). Cronbach's alpha reliability for the IES-R full scale and subscales in this study were as follows: $\mathrm{T} 2$ full scale $=.95, \mathrm{~T} 2$ Intrusion $=.90, \mathrm{~T} 2$ Avoidance $=.84, \mathrm{~T} 2$ Hyperarousal $=.89, \mathrm{~T} 3$ full scale $=.95, \mathrm{~T} 3$ Intrusion $=.91, \mathrm{~T} 3$ Avoidance $=.85$, and T3 Hyperarousal $=.89$.

General psychological distress. The K6 scale, a six-item screening measure of nonspecific psychological distress (Kessler et al., 2003), was used to assess predisaster psychological distress. This scale has been shown to have good psychometric properties (Furukawa, Kessler, Slade, \& Andrews, 2003) and has been used 
in previous research on the psychological functioning of Hurricane Katrina survivors (e.g., Galea et al., 2007). Participants rated items (e.g., "During the past 30 days, about how often did you feel so depressed that nothing could cheer you up?") on a 5-point Likerttype scale ranging from 0 (none of the time) to 4 (all the time). Reliability of the K6 scale in this study was Cronbach's alpha of .70 at $\mathrm{T} 1$.

Hurricane-related stressors. Two variables, both assessed at T2, were included as indicators of hurricane exposure. First, a Hurricane-Related Stressors Scale that included 16 questions assessed stressors experienced during the hurricanes and the week that followed. The questions were drawn from a larger survey of the demographic and health characteristics, evacuation and hurricane experiences, and future plans of Hurricane Katrina evacuees. The Washington Post, the Kaiser Family Foundation, and the Harvard School of Public Health jointly designed the scale (Brodie, Weltzien, Altman, Blendon, \& Benson, 2006). Participants were asked to indicate whether they had experienced the following as a result of the hurricanes: (a) lacked enough fresh water to drink, (b) lacked enough food to eat, (c) felt their life was in danger, (d) lacked necessary medicine, (e) lacked necessary medical care, (f) had a family member who lacked necessary medical care, $(\mathrm{g})$ lacked knowledge of safety of children, and (h) lacked knowledge of safety of other family members. These questions were asked for both Hurricane Katrina and Hurricane Rita, yielding 16 items in total, each with response options Yes (1) and No (0). A composite score with the count of affirmative responses to these items was created (Kuder-Richardson-20 = .84).

Second, a dummy code indicating whether participants had lost a family member or close friend due to the hurricanes and their aftermath (bereavement) was included, as previous research has indicated this as a stressor that increases survivors' likelihood of PTS and PTG (e.g., Gibbs, 1989; Xu \& Liao, 2011).

Optimism. The Life Orientation Test-Revised (LOT-R) is a self-report measure of optimism that consists of six items with demonstrated reliability and validity (Scheier, Carver, \& Bridges, 1994). Each item was rated on a 4-point Likert scale ranging from 1 (strongly disagree) to 4 (strongly agree). Three of the six items were framed positively (e.g., "I am always optimistic about my future"), and the remaining three were framed negatively (e.g., "If something can go wrong for me, it will"). In this study, Cronbach's alpha was .55 at $\mathrm{T} 1$ and .70 at $\mathrm{T} 2$.

Purpose in life. The MacArthur Network on Transitions to Adulthood developed a 13-item Sense of Self measure for the evaluation of the Opening Doors program (Brock \& Richburg Hayes, 2006). The full measure included two subscales: Purpose in Life (seven items, e.g., "You feel your life is filled with meaning, a sense of purpose," "You can envision the type of person you'd like to become") and Relations With Others (e.g., "There is at least one person who knows 'the real you,", "People often seek your advice and support"). The full scale has been shown to be reliable and associated with educational attainment (Scrivener et al., 2008). Because the current study focused on personality characteristics as predictors of PTS and PTG, we chose to include only the Purpose in Life subscale. The seven items were rated on a 4-point Likerttype scale from 1 (strongly disagree) to 4 (strongly agree). Cronbach's alpha of the scale for the current study at .78 at T1 and .84 at $\mathrm{T} 2$.

\section{Results}

\section{Descriptive Analysis}

As indicated previously, the current study included only female participants who completed surveys at each time point $(N=334)$. A series of $t$ tests and chi-square tests, with Bonferroni corrections for multiple tests, revealed no differences between the participants who completed the three assessments and those who did not. For the 334 women included in the study, we also tested for differences between those who were in the experimental and the control groups in the initial study, again using Bonferroni-corrected $t$ tests and chi-square tests. No significant differences were detected. Last, we examined differences between participants for whom we had complete data $(88.9 \%, n=297)$ and those who were missing data on any of the variables included in the current study $(11.1 \%$, $n=37)$. Again, no significant differences were found. Among the variables that we included in this study, the missing rate ranged from $0.0 \%$ to $4.2 \%$, and the overall rate was $0.6 \%$. We conducted multiple imputation using the Amelia II software (Honaker, King, \& Blackwell, 2008) in R to handle missing data, and five complete data sets were then used for statistical analysis. Results represent an average of the five separate analyses with Rubin's (1987) correction of standard error. We replicated our analysis using only the 297 complete cases and the trends in the data persisted. Prior to conducting statistical analyses, the distributions of each variable were carefully examined, and assumptions of normality were met. Means and standard deviations for all variables in the study, as well as a correlation matrix, are listed in Table 1 .

\section{Associations Between PTG and PTS Full Scale and Subscales}

As shown in Table 1, T3 PTG was significantly correlated with T2 and T3 PTS total and the three PTS subscales, with coefficients ranging from .20 to .29 (all $p<.001$ ), such that participants reporting higher PTS total and subscale scores at each point reported higher PTG. Within each time point, there was a trend toward stronger positive correlations between PTG and avoidance, compared with the correlations between PTG and intrusion and hyperarousal symptoms; however, the differences between the correlations did not reach statistical significance. In supplementary regression analyses, we assessed for curvilinear relationships between PTS total and subscales and PTG by first entering linear terms (e.g., T2 PTS total) and then quadratic terms (e.g., T2 PTS total, squared). In no case was the quadratic term significant above and beyond the linear term, indicating a lack of significant curvilinear relationships.

We also examined PTG scores for participants with and without probable PTSD (i.e., IES-R > 33) at each time point through analysis of variance (ANOVA). As shown in Table 2, at both time points, participants with PTSD had significantly higher PTG than those without PTSD. To further delve into the PTS-PTG relationship, we divided participants into low, medium, and high categories on PTS total and subscales. Since the IES-R does not have established cutoffs for levels of PTS severity (e.g., mild, moderate, severe), we based the categories on the T2 assessments (lower level than $1 / 2$ standard deviation below the mean; medium: within $1 / 2$ standard deviation of the mean; and high: greater than $1 / 2$ 


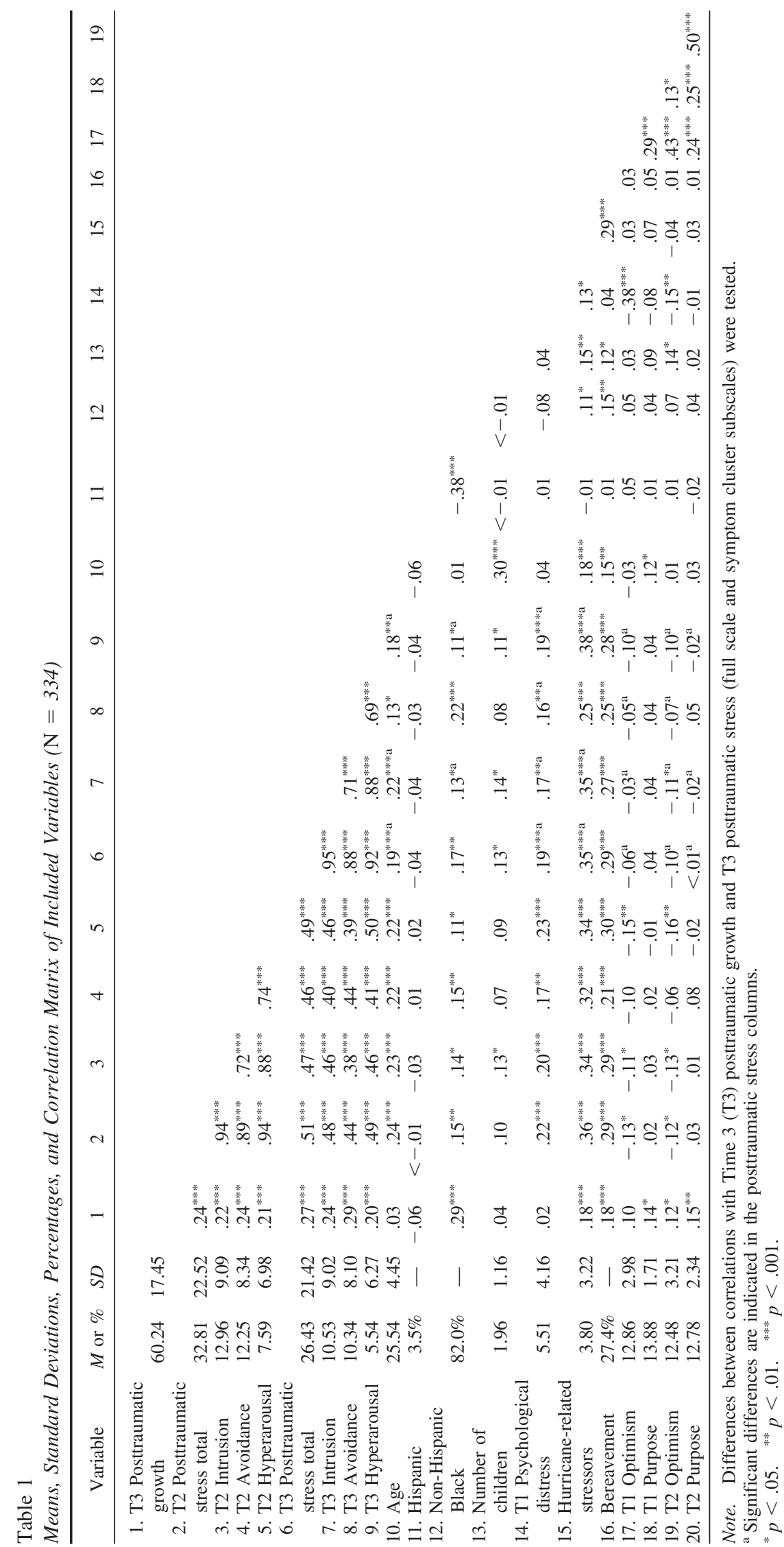


Table 2

Analysis of Variance Examining Group Differences in Time 3 Posttraumatic Growth by Probable PTSD Status, and Levels of PTS Total and Subscales at Times 2 and $3(\mathrm{~N}=334)$

\begin{tabular}{|c|c|c|c|c|c|c|c|c|}
\hline \multirow[b]{2}{*}{ Variable } & \multicolumn{4}{|c|}{ Time 2} & \multicolumn{4}{|c|}{ Time 3} \\
\hline & $N(\%)$ & $M(S D)$ & $F$ & Pairwise comparisons & $N(\%)$ & $M(S D)$ & $F$ & Pairwise comparisons \\
\hline PTSD & & & $12.57^{* * * *}$ & - & & & $11.83^{* * * *}$ & - \\
\hline No & $170(50.9)$ & $56.98(18.05)$ & & & $213(63.8)$ & $57.81(18.05)$ & & \\
\hline Yes & $164(49.1)$ & $64.64(13.82)$ & & & $121(36.2)$ & $64.54(15.51)$ & & \\
\hline PTS Total & & & $10.63^{* * * *}$ & Low $<\operatorname{High}^{* * * *}$ & & & $9.51^{* * * *}$ & Low $<\operatorname{High}^{* * * *}$ \\
\hline Low & $118(35.3)$ & $55.23(20.74)$ & & & $161(48.2)$ & $56.54(18.65)$ & & \\
\hline Medium & $116(34.7)$ & $60.51(15.42)$ & & & $103(30.8)$ & $61.56(16.72)$ & & \\
\hline High & $100(29.9)$ & $65.86(13.34)$ & & & $70(21.0)$ & $66.93(13.00)$ & & \\
\hline Intrusion & & & $9.30^{* * * *}$ & Low $<$ High $^{* * * *}$, Medium ${ }^{* *}$ & & & $8.85^{* * * *}$ & $\begin{array}{l}\text { Low < High } \text { He**; }^{*} \text { Medium } \\
\quad<\text { High* }^{*}\end{array}$ \\
\hline Low & $125(37.4)$ & $55.29(20.78)$ & & & $165(49.4)$ & $57.00(18.50)$ & & \\
\hline Medium & $101(30.2)$ & $61.63(14.86)$ & & & $85(25.4)$ & $60.26(18.06)$ & & \\
\hline High & $108(32.3)$ & $64.69(13.82)$ & & & $84(25.1)$ & $66.62(12.38)$ & & \\
\hline Avoidance & & & $8.58^{* * * *}$ & Low $<\operatorname{High}^{* * * *}$ & & & $12.67^{* * * *}$ & Low $<$ High $^{* * * *}$, Medium ${ }^{* * *}$ \\
\hline Low & $122(36.5)$ & $55.82(19.60)$ & & & $156(46.7)$ & $55.71(18.83)$ & & \\
\hline Medium & $104(31.1)$ & $60.34(15.96)$ & & & $98(29.3)$ & $61.93(18.06)$ & & \\
\hline High & $108(32.3)$ & $65.16(14.89)$ & & & $80(24.04)$ & $66.62(12.38)$ & & \\
\hline Hyperarousal & & & $6.59^{* * *}$ & Low $<\operatorname{High}^{* * * *}$ & & & $6.23^{* * *}$ & Low $<$ High $^{*}$, Medium** \\
\hline Low & $147(44.0)$ & $56.64(20.05)$ & & & $185(55.4)$ & $57.28(18.95)$ & & \\
\hline Medium & $91(27.3)$ & $61.46(14.40)$ & & & $81(24.3)$ & $64.42(13.80)$ & & \\
\hline High & $96(28.7)$ & $64.63(14.58)$ & & & $68(20.4)$ & $63.39(15.65)$ & & \\
\hline
\end{tabular}

Note. $\quad$ PTSD $=$ posttraumatic stress disorder; PTS $=$ posttraumatic stress.

${ }^{*} p<.05 .^{* * *} p<.01$. $^{* * * *} p<.001$.

standard deviation above the mean). The results of ANOVA with Bonferroni-corrected pairwise comparisons indicated that in each case, participants in the high category had significantly higher PTG than those in the low category (Table 2).

\section{Patterns of PTS Over Time and PTG}

Next, we examined how PTG was related to patterns of PTS over time. First, we conducted multiple regression analysis predicting PTG, including main effects for T2 and T3 PTS total, as well as an interaction between PTS total at both time points. PTS total scores were centered prior to the analysis to permit exploration of the interaction. Controlling for main effects, the interaction between T2 and T3 PTS total was marginally significant $(b<$ -0.01 , standard error $[S E]=<0.01, t=-1.72, p=.08)$. Decomposition of the interaction indicated that the relationship between T3 PTS total and PTG was strongest among those with higher T2 PTS total. The same procedure was repeated for the symptom cluster subscales, and the interaction term for avoidance was significant ( $b=-0.02, S E=0.01, t=-2.34, p=.02)$, whereas those for intrusion and hyperarousal were not $(b<-0.01$, $S E=0.01, t=-0.39, p=.70$, and $b=-0.03, S E=0.02, t=$ $-1.58, p=.11)$. Decomposition of the interaction for avoidance indicated the same pattern as for the PTS total.

Next, we examined differences in PTG by probable PTSD classification and PTS total and subscale categories over time using ANOVA and Bonferroni-corrected pairwise comparisons (Table 3). We found that participants with probable PTSD at both time points had significantly higher PTG than those who never had probable PTSD. For the PTS total and subscale categories, we detected several significant differences between participants who had low levels at both T2 and T3 and those who exhibited other patterns, most consistently, participants who had high levels at both time points. Additional significant differences between the categories are listed in Table 3. Notably, there were no significant differences in PTG among the groups that had increasing, decreasing, or consistently high PTS over time.

\section{Predictors of PTG and PTS}

To assess for variation in relationships between demographic, hurricane-related, and personality variables on T3 PTG and T3 PTS total and subscales, we first assessed for significant differences in bivariate correlations (Table 1). Relative to the correlations with PTG, we found significantly stronger positive correlations of PTS variables with age (for PTS total, Intrusion, and Hyperarousal), T1 psychological distress (PTS total and all subscales), and hurricane-related stressors (PTS total, Avoidance). In contrast, PTG had significantly stronger positive correlations with non-Hispanic Black race (vs. Intrusion, Hyperarousal), T1 and T2 optimism (vs. PTS total and all subscales), and T2 purpose (vs. PTS total, Intrusion, Hyperarousal).

Next, hierarchical regression analyses predicting T3 PTG and T3 PTS total and subscales were conducted (Table 4). In Step 1, demographic variables and T1 psychological distress were entered and accounted for significant variance in all four outcomes. NonHispanic Black race was a significant unique predictor of higher PTG, and older age, non-Hispanic Black race, and higher T1 psychological distress were significant unique predictors of higher PTS total and subscales. Neither Hispanic ethnicity nor number of children was a significant predictor in any of the models. In Step 2 , hurricane-related variables were entered and accounted for significant additional variance in all four outcomes. Exposure to hurricane-related stressors and bereavement were both signifi- 


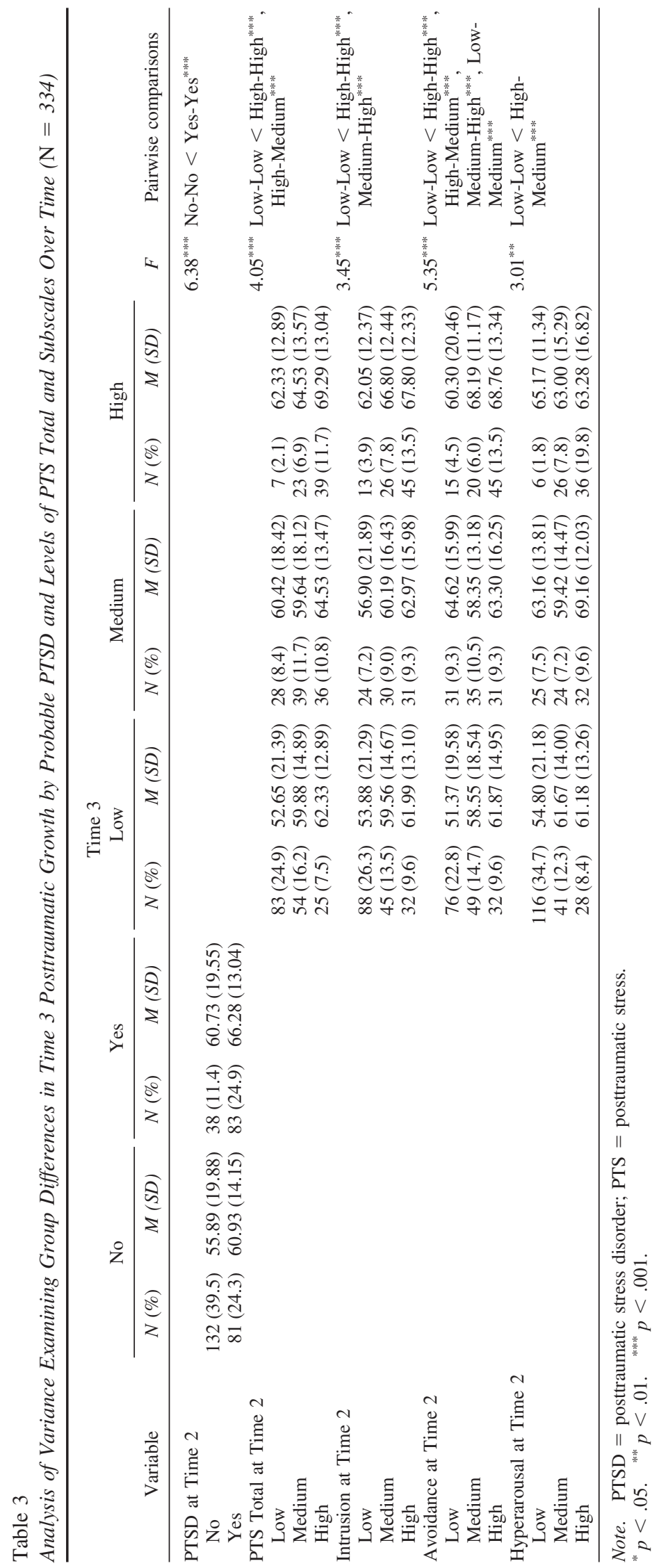


Table 4

Hierarchical Regression Models Predicting Time 3 Posttraumatic Growth and Posttraumatic Stress Total and Subscales (N = 334)

\begin{tabular}{|c|c|c|c|c|c|c|c|c|c|c|}
\hline \multirow[b]{2}{*}{ Variable } & \multicolumn{5}{|c|}{ T3 Posttraumatic growth } & \multicolumn{5}{|c|}{ T3 Posttraumatic total } \\
\hline & $b$ & $S E$ & $t$ & $R^{2}\left(R^{2} \Delta\right)$ & $F(F \Delta)$ & $b$ & $S E$ & $t$ & $R^{2}\left(R^{2} \Delta\right)$ & $F(F \Delta)$ \\
\hline Step 1. Baseline characteristics & & & & .09 & $6.35^{* * *}$ & & & & .11 & $8.08^{* * * *}$ \\
\hline Age & 0.07 & .22 & 0.33 & & & 0.81 & 0.27 & $3.06^{* *}$ & & \\
\hline Hispanic & 5.24 & 5.42 & 0.97 & & & 4.69 & 6.57 & 0.71 & & \\
\hline Non-Hispanic Black & 14.10 & 2.60 & $5.42^{* * * *}$ & & & 11.20 & 3.16 & $3.55^{* * * *}$ & & \\
\hline Number of children & 0.44 & 0.83 & 0.53 & & & 1.26 & 1.01 & 1.25 & & \\
\hline T1 Psychological distress & 0.19 & 0.22 & 0.83 & & & 0.99 & 0.27 & $3.68^{* * * *}$ & & \\
\hline Step 2. Hurricane exposure & & & & $.12(.03)$ & $6.34^{* * * *}\left(5.85^{* *}\right)$ & & & & $.21(.10)$ & $12.54^{* * * *}\left(21.21^{* * * *}\right)$ \\
\hline Hurricane-related stressors & 0.65 & 0.30 & $2.16^{*}$ & & & 1.62 & 0.35 & $4.62^{* * * *}$ & & \\
\hline Bereavement & 4.39 & 2.16 & $2.03^{*}$ & & & 8.25 & 2.51 & $3.29 * * * *$ & & \\
\hline Step 3. T1 psychological resources & & & & $.14(.02)$ & $5.81^{* * * *}\left(3.61^{*}\right)$ & & & & $.21(<.01)$ & $9.73^{* * * *}(.12)$ \\
\hline 3a T1 Optimism & 0.62 & 0.33 & 1.87 & $.13(.01)$ & $6.03^{* * *}(3.51)$ & -0.14 & 0.39 & -0.36 & $.21(<.01)$ & $10.96^{* * * *}(.13)$ \\
\hline 3b T1 Purpose & 1.27 & 0.54 & $2.37^{*}$ & $.13(.01)$ & $6.32^{* * *}\left(5.60^{*}\right)$ & 0.13 & 0.63 & 0.21 & $.21(<.01)$ & $10.95^{* * * *}(.05)$ \\
\hline Step 4. T2 psychological resources & & & & $.15(.01)$ & $5.20^{* * * *}(2.25)$ & & & & $.22(.01)$ & $8.36^{* * * *}(1.93)$ \\
\hline 4a T2 Optimism & 0.49 & 0.32 & 1.54 & $.14(.01)$ & $5.65^{* * * *}(2.39)$ & -0.68 & 0.37 & -1.84 & $.22(.01)$ & $10.20^{* * * *}(3.41)$ \\
\hline 4b T2 Purpose & 0.87 & 0.40 & $2.21^{*}$ & $.15(.01)$ & $6.24^{* * *}\left(4.92^{*}\right)$ & -0.16 & 0.47 & -0.34 & $.22(.01)$ & $9.72^{* * * *}(.11)$ \\
\hline
\end{tabular}

Note. $\mathrm{T} 1=$ Time $1 ; \mathrm{T} 2=$ Time $2 ; \mathrm{T} 3=$ Time 3.

${ }^{*} p<.05 . \quad{ }^{* * *} p<.01 .^{* * *} p<.001$.

cantly and uniquely predictive of higher PTG and PTS total and subscales.

In supplementary analyses, we entered $\mathrm{T} 1$ psychological resources in Step 3 and T2 psychological resources in Step 4. We note here that because $\mathrm{T} 1$ levels were controlled, the analyses permit insight into how changes in psychological resources from pre- to postdisaster predict PTG and PTS total and subscales. The results of these analyses were inconsistent with those in the bivariate analyses, and because we suspected confounding between optimism and purpose, we tested separate analyses for each psychological resource. Predisaster optimism was not significant in any of the models. Predisaster purpose was a significant predictor of PTG, such that participants with higher T1 purpose had higher PTG, whereas predisaster purpose was not a significant predictor of PTS total or subscales. Postdisaster optimism accounted for significant additional variance in Intrusion, such that participants with lower T2 optimism had higher T3 Intrusion. In contrast, postdisaster optimism did not account for significant additional variance in PTG, PTS total, avoidance, or hyperarousal. Postdisaster purpose accounted for significant additional variance in PTG, with higher PTG among participants with higher T2 purpose. Again, T2 purpose did not account for significant additional variance in PTS total or subscales.

\section{Discussion}

The primary purpose of this study was to provide greater clarity into the relationship between posttraumatic growth (PTG) and posttraumatic stress (PTS) among low-income mothers who were exposed to Hurricane Katrina. We found that PTS, assessed at both 1 and 3 years after Hurricane Katrina, was significantly and positively associated with PTG assessed at 3 years postdisaster. This was the case for total PTS and for the three PTS symptom clusters. Among the PTS symptom clusters, Avoidance was most strongly associated with PTG, perhaps indicative of illusory growth; however, differences among the correlations between PTS symptom clusters and PTG did not reach statistical significance.
Additionally, survivors with probable PTSD at each time point, as indicated by clinical cutoff scores, as well as those with consistently high levels of PTS total and symptom clusters, reported significantly higher levels of PTG. These results suggest that experiencing higher levels of PTS, whether in the more immediate or longer term aftermath of disasters, is associated with higher PTG. These results are also consistent with the Janus Face model of PTG (Maercker \& Zoellner, 2004) in that PTG was related to aspects of PTS that are thought to indicate both functional growth (intrusion) and illusory growth (avoidance, hyperarousal).

Because PTS was assessed at both postdisaster time points, our analyses also shed light into how patterns of PTS over time might yield different levels of PTG. To address this issue, we first computed an interaction of PTS total at both postdisaster time points and found that, controlling for main effects, the interaction was a marginally significant predictor of PTG. We replicated these analyses with the PTS subscales and found that the interaction term was significant for Avoidance, but not for Intrusion or Hyperarousal. The results here suggested that survivors who sustained high levels of avoidance over time were most likely to report PTG.

However, when we investigated patterns of PTS over time using categorical variables, we found that PTG was consistently highest among survivors who had high levels of PTS, both total and symptom clusters, over time. Using the clinical cutoffs to categorize survivors into patterns of probable PTSD over time, we found that survivors who were consistently classified as having probable PTSD had significantly higher PTG than those who never surpassed the cutoff for probable PTSD. Looking at categories of low, medium, and high PTS total and symptom clusters, we found that participants with low PTS at both postdisaster time points had significantly lower PTG than those who exhibited other patterns, most consistently, the participants who had high PTS at both time points. We also noted that there were no significant differences in PTG between any of the other PTS total or subscale patterns. That is, survivors whose PTS increased, decreased, or was consistently high did not have significantly different levels of PTG. Future 


\begin{tabular}{|c|c|c|c|c|c|c|c|c|c|c|c|c|c|}
\hline \multicolumn{5}{|c|}{$\mathrm{T} 3$ Intrusion } & \multicolumn{5}{|c|}{ T3 Avoidance } & \multicolumn{4}{|c|}{ T3 Hyperarousal } \\
\hline$b$ & $S E$ & $t$ & $R^{2}\left(R^{2} \Delta\right)$ & $F(F \Delta)$ & $b$ & $S E$ & $t$ & $R^{2}\left(R^{2} \Delta\right)$ & $F(F \Delta)$ & $b$ & $S E$ & $t$ & $R^{2}\left(R^{2} \Delta\right)$ \\
\hline & & & \multirow{6}{*}{.10} & \multirow{6}{*}{$7.23^{* * * *}$} & & & & \multirow{6}{*}{.10} & \multirow{6}{*}{$7.55^{* * * *}$} & & & & \multirow[t]{6}{*}{.08} \\
\hline 0.38 & 0.11 & $3.40^{* * * * *}$ & & & 0.21 & 0.10 & $2.14^{*}$ & & & 0.21 & 0.08 & $2.71^{*}$ & \\
\hline 1.14 & 2.78 & 0.41 & & & 3.15 & 2.49 & 1.26 & & & 0.39 & 1.95 & 0.20 & \\
\hline 3.53 & 1.34 & $2.64^{* *}$ & & & 5.56 & 1.20 & $4.64^{* * * *}$ & & & 2.12 & 0.94 & $2.26^{*}$ & \\
\hline 0.63 & 0.43 & 1.47 & & & 0.30 & 0.38 & 0.79 & & & 0.33 & 0.30 & 1.10 & \\
\hline 0.37 & 0.11 & $3.21^{* *}$ & & & 0.33 & 0.10 & $3.25^{* *}$ & & & 0.29 & 0.08 & $3.65^{* * * *}$ & \\
\hline & & & \multirow[t]{3}{*}{$.20(.10)$} & \multirow[t]{3}{*}{$11.39^{* * * *}\left(19.71^{* * * *}\right)$} & & & & \multirow[t]{3}{*}{$.16(.06)$} & \multirow[t]{3}{*}{$8.65^{* * * *}\left(10.32^{* * * *}\right)$} & & & & \multirow[t]{3}{*}{$.21(.13)$} \\
\hline 0.68 & 0.15 & $4.56^{\text {***** }}$ & & & 0.38 & 0.14 & $2.78^{* * *}$ & & & 0.56 & 0.10 & $5.42^{* * * *}$ & \\
\hline 3.25 & 1.07 & $3.04^{* * *}$ & & & 2.73 & 0.98 & $2.78^{* *}$ & & & 2.28 & 0.74 & $3.09^{* *}$ & \\
\hline & & & $.20(<.01)$ & $8.81^{\text {**** }}(0.98)$ & & & & $.16(<.01)$ & $6.71^{* * *}(0.09)$ & & & & $.21(<.01)$ \\
\hline 0.03 & 0.16 & 0.15 & $.20(<.01)$ & $9.94^{* * * *}(0.02)$ & -0.04 & 0.15 & -0.24 & $.16(<.01)$ & $7.55^{* * * *}(0.06)$ & -0.13 & 0.11 & -1.12 & $.21(<.01)$ \\
\hline 0.04 & 0.27 & 0.16 & $.20(<.01)$ & $9.94^{* * *}(0.03)$ & 0.06 & 0.25 & 0.25 & $.16(<.01)$ & $7.55^{* * *}(0.06)$ & 0.03 & 0.18 & 0.16 & $.21(<.01)$ \\
\hline & & & $.21(.01)$ & $7.76^{* * * *}(2.64)$ & & & & $.17(.01)$ & $5.88^{* * * *}(1.99)$ & & & & $.21(<.01)$ \\
\hline-0.36 & 0.16 & $-2.28^{*}$ & $.21(<.01)$ & $9.54^{* * *}\left(5.21^{*}\right)$ & -0.20 & 0.14 & -1.37 & $.16(<.01)$ & $6.94^{* * * *}(1.88)$ & -0.13 & 0.11 & -1.16 & $.21(<.01)$ \\
\hline-0.15 & 0.20 & -0.74 & & $8.88^{* * * *}(0.55)$ & 0.10 & 0.18 & 0.56 & $.16(<.01)$ & $6.73^{* * * *}(0.32)$ & -0.11 & 0.14 & -0.82 & $.21(<.01)$ \\
\hline
\end{tabular}

studies should further explore how the various patterns of PTS over time relate to PTG, as well as the subtle variations in patterns among the PTS subscales. Additionally, future studies with larger samples would be useful in further unpacking the PTS-PTG relationship.

We found that survivors who identified as non-Hispanic Black reported significantly higher levels of both PTS (total and symptom clusters) and PTG. Higher PTS among non-Hispanic Black women is consistent with prior research (e.g., Brewin et al., 2000) and could relate to the disproportionate damage from the hurricane in non-Hispanic Black communities in New Orleans (e.g., Logan, 2006), to experiences of racism during the hurricane and its aftermath (e.g., White, Philpot, Wylie, \& McGowen, 2007), or to greater difficulties finding suitable housing and employment in postdisaster neighborhoods (Henrici, Helmuth, \& Fernandes, 2010; Liu \& Plyer, 2010). Conversely, strong social ties and religiosity within communities might lead non-Hispanic Black women to experience greater PTG, particularly in the relationship and spirituality domains (e.g., Wilson \& Boden, 2008). We note here that Hispanic ethnicity was not a significant predictor of PTG or PTS (total or symptom clusters). However, given the small percentage of Hispanic women in the sample (3.5\%), this finding should be interpreted with caution, and the postdisaster psychological experiences of this subgroup should be explored in further studies.

We also found that older women were more likely to experience PTS (total and symptom clusters), a finding that is inconsistent with previous findings showing heightened PTS risk among younger participants. It is important to note, however, that because all of the participants were mothers of young children at baseline, the age range in our sample at baseline was restricted to those 18-34 years old. Reconciling our divergent finding, a comprehensive review of disaster research found that, although the majority of studies show that PTS risk declines with age, those that classified adults into younger, middle-aged, and older adults found the highest levels of PTS among the middle-aged (Norris et al., 2002).
Middle adulthood might be associated with other variables that significantly increased PTS risk, a possibility that could be explored in future research. For example, middle-aged women might be more likely to have school-age, versus younger, children, who are more likely to face postdisaster psychosocial difficulties, such as behavioral problems and difficulties adjusting to new schools (Terranova, Boxer, \& Morris, 2009). Number of children was not a significant predictor of PTS or PTG in the current study, and perhaps future research should simultaneously account for the number and age of children in postdisaster households.

Because we had access to predisaster data, we were able to explore how general psychological distress prior to the disaster related to later PTS and PTG. Consistent with prior research linking pre- and postdisaster psychological functioning (e.g., Weems et al., 2007), we found that women with higher predisaster distress had significantly higher postdisaster PTS (total and symptom clusters). This finding underscores the vulnerability of individuals already suffering from mental health difficulties to postdisaster psychological adversity. In contrast, predisaster distress was not a significant predictor of PTG. Although future research is needed, it appears that predisaster distress does not lead directly to higher PTG but could instead yield PTG indirectly through its positive association with PTS.

When variables related to hurricane exposure were then entered into the model, hurricane-related stressors and bereavement significantly predicted both PTS (total and symptom clusters) and PTG. In examining the regression weights, we noted that the composite variable of hurricane-related stressors was a stronger predictor of PTS than PTG and that differences in the correlations were significant (higher for PTS total, Intrusion, and Hyperarousal vs. PTG). The results suggest that some degree of exposure might be necessary for both PTS and PTG to manifest but that the dose-response relationship between exposure and PTS is more pronounced. More nuanced measures of exposure would provide greater insight into whether specific experiences or losses are likely to yield PTS or PTG. 
Next, we explored the role of psychological resources in shaping PTS and PTG. Again, predisaster data allowed for identification of characteristics prior to the disaster that influenced postdisaster responses. We found that participants with a stronger predisaster sense of purpose reported significantly higher PTG. Predisaster purpose could represent an underlying tendency to be actively engaged in one's life and to focus on long-term goals in the face of stressors. Survivors with these characteristics might have then been more likely to engage in cognitive processing to understand their trauma and to actively cope with stressors in the aftermath of disasters, potentially leading to functional growth (Pooley, Cohen, O'Connor, \& Taylor, 2012; Triplett, Tedeschi, Cann, Calhoun, \& Reeve, 2012). Survivors with a stronger sense of purpose might have also been more persistent in pursuing postdisaster resources, such as Federal Emergency Management Agency (FEMA) and insurance claims, and more motivated in their education and careers (Pizzolato, Brown, \& Kanny, 2011). A greater sense of purpose may also result from other resources, including strong social support or religiosity (French \& Joseph, 1999; Krause, 2007), which could promote PTG. Notably, predisaster purpose was not a significant predictor of PTS total or subscales in this study, indicating that the influence of this resource was specific to PTG. Predisaster optimism predicted neither PTG nor PTS (total or subscales), which was contrary to our expectations.

Psychological resources assessed 1 year postdisaster were subsequently entered. It is important to note here that purpose and optimism, although sometimes conceptualized as stable personality characteristics (e.g., Scheier \& Carver, 1985), can change over time and in response to trauma exposure (e.g., Burger \& Palmer, 1992). Because we controlled for predisaster purpose and optimism, we were able to explore how pre- to postdisaster changes in these resources were related to PTS and PTG. Postdisaster optimism was a significant negative predictor of intrusive symptoms, controlling for predisaster optimism. Further research is needed to provide understanding of how declines in optimism relate to intrusion. One possibility is that survivors ruminate on their diminished perceived likelihood of achieving valued personal and family goals due to postdisaster challenges. In contrast, postdisaster optimism was not a predictor of PTS total, Avoidance, or Hyperarousal scores, nor of PTG. It therefore seems that in this case, postdisaster increases in optimism did not facilitate growth.

We also found that higher postdisaster purpose significantly predicted higher PTG, suggesting that changes in purpose relate to the development of PTG. A greater sense of purpose could result from deliberate cognitive processing, which previous research has found to be predictive of PTG (Triplett et al., 2012). Increases in purpose could also be derived from concrete improvements in the aftermath of disaster, such as stronger support networks and enhanced economic prospects, or from involvement in community services. Again, the influence of purpose was specific to PTG, as postdisaster purpose was not significantly associated with PTS total or subscales.

\section{Implications}

The results of the study have implications for research, policy, and practice. Additional longitudinal studies that include multiple assessments of psychosocial resource variables, PTS total and symptom clusters, and PTG would further researchers' understand- ing of how PTG and PTS relate to each other over time. Within this context, researchers should explore additional factors that might help to explain the associations between psychosocial resources and outcomes, for example, coping styles or involvement in education, careers, religion, and community service. Replication of the results is also needed given that the effect sizes for some of our findings were modest, especially those regarding relationships between psychological resources, PTS, and PTG.

Particularly in light of the persistent PTS among many survivors, these results highlight the need for disaster preparedness efforts that minimize exposure to hurricane-related stressors, including plans for evacuation via public transportation, guidelines for what conditions necessitate a mandatory evacuation, and means for enforcing such mandates. Policies that ensure that adequate medical care and necessary medications are available during disasters might also prevent disaster-related fatalities and the psychological toll of bereavement. Efforts in the aftermath of disasters to connect survivors with affordable mental health services, particularly survivors with pre-existing mental health conditions and those who have faced high levels of hurricane exposure, would help to prevent posttraumatic stress reactions.

Once affected survivors are identified, mental health practitioners should strive to employ culturally sensitive and empirically supported interventions to reduce PTS, including cognitive behavioral therapy (CBT) and prolonged exposure therapy (Hobfoll, Watson, et al., 2007). Meaning-making interventions could also enhance survivors' sense of optimism (Lee, Cohen, Edgar, Laizner, \& Gagnon, 2006), potentially alleviating intrusive symptoms. It is less clear whether practitioners should encourage PTG in trauma survivors. Although there is some evidence that successful CBT could lead to meaningful change in PTG (Knaevelsrud, Liedl, \& Maercker, 2010; Zoellner, Rabe, Karl, \& Maercker, 2011), less is known about its relation to indices of psychological health or behavioral changes. As Zoellner et al. (2011) noted, however, the body of literature suggests that increases in PTG can occur independent of declines in distress, perhaps reflective of illusory growth. There are also no treatment studies to our knowledge that focus on disaster survivors and include measures of both PTG and PTS, leaving it to speculation how the two constructs might relate to each other over time in postdisaster clinical interventions. Clearly, more research is needed in this area.

\section{Limitations and Conclusions}

The current study has several notable limitations. First, PTG was not measured at the first postdisaster assessment, and we were therefore unable to explore how changes in PTG relate to PTS over time. A more general limitation is that our first postdisaster assessment did not occur until 12 months after Hurricane Katrina. Therefore, the results do not capture psychological functioning in the immediate aftermath of the disaster. The results also do not provide insight into whether self-reported PTG was functional or illusory, and the circumstances that might give rise to each. Further research that assesses both self-reported PTG and behavioral changes, for example, involvement in community service or improved communication in relationships, as well as PTS total and symptom clusters, is needed.

Additionally, although our predisaster mental health measure, the K6 scale of nonspecific psychological distress, permitted a 
control for predisaster psychological functioning, we did not include a predisaster measure of PTS, nor did we assess exposure to additional traumatic events in either the pre- or postdisaster periods. Inclusion of these variables would have provided greater insight into the role of prior experience with trauma on the development and maintenance of PTS and PTG. Likewise, the hurricane-related stressors variable was confined to the weeks during which Hurricanes Katrina and Rita occurred, and experiences of loss, deprivation, and life threat beyond this period could account for additional variance in psychological functioning. The measure also did not capture other elements of Hurricane Katrina that could have made the women more or less prone to PTS or PTG, such as property damage and loss, sexual assault, long-term separation from friends and family, or receipt of various forms of assistance from FEMA and nonprofit organizations. Despite previous research indicating its validity and reliability (Scheier et al., 1994), the measure of optimism, the Life Orientation Test, had relatively low reliability at the predisaster assessment. Last, although the inclusion of low-income mothers, primarily nonHispanic Black, allowed for investigation of predictors of PTS and PTG in a sample that was highly vulnerable to hurricane exposure and postdisaster adversity, it limited the extent to which the findings generalize to other populations. Moreover, since the participants were all community college students at baseline, they are not representative of all low-income mothers from the New Orleans area.

Despite its limitations, this study provides greater insight into the PTG-PTS relationship. Consistent with previous research, our results suggest the possibility of personal transformation in those suffering from the most severe traumatic reactions to disaster. Particularly when equipped with a sense a purpose, the experience of disaster-related trauma appears to open some survivors to an improved sense of life's possibilities. PTG and PTS are not entirely overlapping, however, and a range of factors differentially predicts the likelihood of each. To the extent that research continues to uncover the conditions and mechanisms that give rise to growth, we will be better positioned to tip the balance toward PTG when the next natural disaster inevitably strikes.

\section{References}

Ahmad, S., Feder, A., Lee, E. J., Wang, Y., Southwick, S. M., Schlackman, E., . . . Charney, D. S. (2010). Earthquake impact in a remote South Asian population: Psychosocial factors and posttraumatic symptom. Journal of Traumatic Stress, 23, 408-412. doi:10.1002/jts.20535

Bonanno, G. A. (2004). Loss, trauma, and human resilience: Have we underestimated the human capacity to thrive after extremely aversive events? American Psychologist, 59, 20-28. doi:10.1037/0003-066X.59 .1 .20

Bostock, L., Shiekh, A. I., \& Barton, S. (2009). Posttraumatic growth and optimism in health-related trauma: A systemic review. Journal of Clinical Psychology in Medical Settings, 16, 281-296. doi:10.1007/s10880009-9175-6

Brewin, C. R., Andrews, B., \& Valentine, J. D. (2000). Meta-analysis of risk factors for posttraumatic stress disorder in trauma-exposed adults. Journal of Consulting and Clinical Psychology, 68, 748-766. doi: 10.1037/0022-006X.68.5.748

Brock, T., \& Richburg-Hayes, L. (2006). Paying for persistence: Early results of a Louisiana scholarship program for low-income parents attending community college. New York, NY: MDRC.
Brodie, M., Weltzien, E., Altman, D., Blendon, R. J., \& Benson, J. M. (2006). Experiences of Hurricane Katrina evacuees in Houston shelters: Implications for future planning. American Journal of Public Health, 96, 1402-1408. doi:10.2105/AJPH.2005.084475

Burger, J. M., \& Palmer, M. L. (1992). Changes in and generalization of unrealistic optimism following experiences with stressful events: Reactions to the 1989 California earthquake. Personality and Social Psychology Bulletin, 18, 39-43. doi:10.1177/0146167292181006

Butler, L. D., Blasey, C. M., Garlan, R. W., McCaslin, S. E., Azarow, J., Chen, X. H., . . Hastings, T. A. (2005). Posttraumatic growth following the terrorist attacks of September 11, 2001: Cognitive, coping, and trauma symptom predictors in an Internet convenience sample. Traumatology, 11, 247-267. doi:10.1177/153476560501100405

Creamer, M., Bell, R., \& Failla, S. (2003). Psychometric properties of the Impact of Event Scale-Revised. Behaviour Research and Therapy, 41, 1489-1496. doi:10.1016/j.brat.2003.07.010

Dekel, S., Ein-Dor, T., \& Solomon, Z. (2012). Posttraumatic growth and posttraumatic distress: A longitudinal study. Psychological Trauma: Theory, Research, Practice, and Policy, 4, 94-101. doi:10.1037/ a0021865

Elliott, J. R., \& Pais, J. (2006). Race, class, and Hurricane Katrina: Social differences in human response to disaster. Social Science Research, 35, 295-321. doi:10.1016/j.ssresearch.2006.02.003

French, S., \& Joseph, S. (1999). Religiosity and its association with happiness, purpose in life, and self-actualisation. Mental Health, Religion \& Culture, 2, 117-120. doi:10.1080/13674679908406340

Furukawa, T. A., Kessler, R. C., Slade, T., \& Andrews, G. (2003). The performance of the K6 and K10 Screening Scales for psychological distress in the Australian National Survey of Mental Health and WellBeing. Psychological Medicine, 33, 357-362. doi:10.1017/ S0033291702006700

Galea, S., Brewin, C. R., Gruber, M., Jones, R. T., King, D. W., King, L. A., .. Kessler, R. C. (2007). Exposure to hurricane-related stressors and mental illness after Hurricane Katrina. Archives of General Psychiatry, 64, 1427-1434. doi:10.1001/archpsyc.64.12.1427

Galea, S., Tracy, M., Norris, F., \& Coffey, S. F. (2008). Financial and social circumstances and the incidence and course of PTSD in Mississippi during the first two years after Hurricane Katrina. Journal of Traumatic Stress, 21, 357-368. doi:10.1002/jts.20355

Gibbs, M. S. (1989). Factors in the victim that mediate between disaster and psychopathology: A review. Journal of Traumatic Stress, 2, 489514. doi: $10.1002 /$ jts. 2490020411

Henrici, J. M., Helmuth, A. S., \& Fernandes, R. (2010). Mounting losses: Women and public housing after Hurricane Katrina. Washington, DC: Institute for Women's Policy Research.

Hobfoll, S. E., Hall, B. J., Canetti-Nisim, D., Galea, S., Johnson, R. J., \& Palmieri, P. A. (2007). Refining our understanding of traumatic growth in the face of terrorism: Moving from meaning cognitions to doing what is meaningful. Applied Psychology, 56, 345-366. doi:10.1111/j.14640597.2007.00292.x

Hobfoll, S. E., Watson, P., Bell, C. C., Bryan, R. A., Brymer, M. J., Friedman, M. J., . . Ursano, R. J. (2007). Five essential elements of immediate and mid-term mass trauma intervention: Empirical evidence. Psychiatry: Interpersonal and Biological Processes, 70, 283-315. doi: 10.1521/psyc.2007.70.4.283

Honaker, J., King, G., \& Blackwell, M. (2008). Amelia II: A program for missing data [Computer software]. Retrieved June 15, 2009, from http:// gking.harvard.edu/amelia/

Kessler, R. C., Barker, P. R., Colpe, L. J., Epstein, J. F., Groerer, J. C., Hiripi, E., . . Zaslavsky, A. M. (2003). Screening for serious mental illness in the general population. Archives of General Psychiatry, 60, 184-189. doi:10.1001/archpsyc.60.2.184

Kimhi, S., \& Eshel, Y., Zysberg, L., \& Hantman, S. (2010). Postwar winners and losers in the long run: Determinants of war related stress 
symptoms and posttraumatic recovery. Community Mental Health Journal, 46, 10-19. doi:10.1007/s10597-009-9183-x

Knabb, R. D., Rhome, J. R., \& Brown, D. P. (2006). Tropical cyclone report: Hurricane Katrina, 23-30 August 2005. Retrieved from http:// nhc.noaa.gov/pdf/TCR-AL122005_Katrina.pdf

Knaevelsrud, C., Liedl, A., \& Maercker, A. (2010). Posttraumatic growth, optimism, and openness as outcomes of a cognitive-behavioral intervention for posttraumatic stress reactions. Journal of Health Psychology, 15, 1030-1038. doi:10.1177/1359105309360073

Krause, N. (2007). Longitudinal study of social support and meaning in life. Psychology and Aging, 22, 456-469. doi:10.1037/0882-7974.22.3 .456

Lee, V., Cohen, S. R., Edgar, L., Laizner, A. M., \& Gagnon, A. J. (2006). Meaning-making intervention during breast or colorectal cancer treatment improves self-esteem, optimism, and self-efficacy. Social Science \& Medicine, 62, 3133-3145. doi:10.1016/j.socscimed.2005.11.041

Lindstrom, C. M., Cann, A., Calhoun, L. G., \& Tedeschi, R. G. (2013). The relationship of core belief challenge, rumination, disclosure, and sociocultural elements to posttraumatic growth. Psychological Trauma: Theory, Research, Practice, and Policy, 5, 50-55. doi:10.1037/a0022030

Linley, P. A., \& Joseph, S. (2004). Positive change following trauma and adversity: A review. Journal of Traumatic Stress, 17, 11-21. doi: 10.1023/B:JOTS.0000014671.27856.7e

Linley, P. A., \& Joseph, S. (2011). Meaning in life and posttraumatic growth. Journal of Loss and Trauma, 16, 150-159. doi:10.1080/ 15325024.2010.519287

Liu, A. k., \& Plyer, A. (2010). An overview of Greater New Orleans: From recovery to transformation. In The New Orleans Index at Five (pp. 1-20). Washington, DC: Brookings Institution and Greater New Orleans Community Data Center.

Logan, J. R. (2006). The impact of Katrina: Race and class in stormdamaged neighborhoods. Unpublished manuscript. Spatial Structures in the Social Sciences Initiative, Brown University, Providence, RI.

Maercker, A., \& Zoellner, T. (2004). The Janus face of self-perceived growth: Toward a two-component model of posttraumatic growth. Psychological Inquiry, 15, 41-48.

Norris, F., Friedman, M., Watson, P., Byrne, C., Diaz, E., \& Kaniasty, K. (2002). 60,000 disaster victims speak. Part I: An empirical review of the empirical literature, 1981-2001. Psychiatry, 65, 207-239. doi:10.1521/ psyc.65.3.207.20173

Owens, G. P., Steger, M. F., Whitesell, A. A., \& Herrera, C. J. (2009). Posttraumatic stress disorder, guilt, depression, and meaning in life among military veterans. Journal of Traumatic Stress, 22, 654-657. doi: $10.1002 /$ jts. 20460

Pizzolato, J. E., Brown, E. L., \& Kanny, M. A. (2011). Purpose plus: Supporting youth purpose, control, and academic achievement [Special issue]. New Directions for Youth Development, 2011, 75-88. doi: $10.1002 / y d .429$

Pooley, J. A., Cohen, L., O'Connor, M., \& Taylor, M. (2012). Posttraumatic stress and posttraumatic growth and their relationship to coping and self-efficacy in Northwest Australian Cyclone Communities. Psychological Trauma: Theory, Research, Practice, and Policy. Advance online publication. doi:10.1037/a0028046

Prati, G., \& Pietrantoni, L. (2009). Optimism, social support, and coping strategies as factors contributing to posttraumatic growth: A metaanalysis. Journal of Loss and Trauma, 14, 364-388. doi:10.1080/ 15325020902724271

Richburg Hayes, L., Brock, T., LeBlanc, A., Paxson, C., Rouse, C. E., \& Barrow, L. (2009). Rewarding persistence: Effects of a performancebased scholarship program for low-income parents. New York, NY: MDRC. doi:10.2139/ssrn.1353360

Rubin, D. B. (1987). Multiple imputation for nonresponse in surveys. New York, NY: Wiley. doi:10.1002/9780470316696
Scheier, M. F., \& Carver, C. S. (1985). Optimism, coping, and health Assessment and implications of generalized outcome expectancies. Health Psychology, 4, 219-247. doi:10.1037/0278-6133.4.3.219

Scheier, M. F., Carver, C. S., \& Bridges, M. W. (1994). Distinguishing optimism from neuroticism (and trait anxiety, self-mastery and selfesteem): A reevaluation of the Life Orientation Test. Journal of Personality and Social Psychology, 67, 1063-1078. doi:10.1037/0022-3514.67 .6 .1063

Scrivener, S., Bloom, D., LeBlanc, A., Paxson, C., Rouse, C. E., \& Sommo, C. (2008). A good start: Two-year effects of a freshman learning community program at Kingsborough Community College. New York, NY: MDRC.

Shigemoto, Y., \& Poyrazli, S. (in press). Factors related to posttraumatic growth in U.S. and Japanese college students. Psychological Trauma: Theory, Research, Practice, and Policy.

Solomon, Z., \& Dekel, R. (2007). Posttraumatic stress disorder and posttraumatic growth among Israeli ex-POWs. Journal of Traumatic Stress, 20, 303-312. doi: $10.1002 /$ jts. 20216

Stockton, H., Hunt, N., \& Joseph, S. (2011). Cognitive processing, rumination, and posttraumatic growth. Journal of Traumatic Stress, 24, 85-92. doi:10.1002/jts.20606

Taku, K., Cann, A., Tedeschi, R. G., \& Calhoun, L. G. (2009). Intrusive versus deliberate rumination in posttraumatic growth across U.S. and Japanese samples. Anxiety, Stress \& Coping, 22, 129-136. doi:10.1080/ 10615800802317841

Tang, C. S. (2007). Posttraumatic growth of Southeast Asian survivors with physical injuries six months after the 2004 Southeast Asian earthquake-tsunami. Australian Journal of Disaster and Traumatic Studies. Retrieved fromwww.massey.ac.nz/ trauma/

Tedeschi, R. G., \& Calhoun, L. G. (1995). Trauma and transformation: Growing in the aftermath of suffering. Thousand Oaks, CA: Sage.

Tedeschi, R. G., \& Calhoun, L. G. (1996). The posttraumatic growth inventory: Measuring the positive legacy of trauma. Journal of Traumatic Stress, 9, 455-472. doi:10.1002/jts.2490090305

Terranova, A. M., Boxer, P., \& Morris, A. S. (2009). Factors influencing the course of posttraumatic stress following a natural disaster: Children's reactions to Hurricane Katrina. Journal of Applied Developmental Psychology, 30, 344-355. doi:10.1016/j.appdev.2008.12.017

Tomich, P. L., \& Helgeson, V. S. (2012). Posttraumatic growth following cancer: Links to quality of life. Journal of Traumatic Stress, 25, 567573. doi: $10.1002 /$ jts. 21738

Triplett, K. N., Tedeschi, R. G., Cann, A., Calhoun, L. G., \& Reeve, C. L. (2012). Posttraumatic growth, meaning in life, and life satisfaction in response to trauma. Psychological Trauma: Theory, Research, Practice, and Policy, 4, 400-410. doi:10.1037/a0024204

U.S. Department of Commerce. (2006). Gulf Coast recovery: 17 months after the hurricanes. Retrieved from Www. economics. noaa. gov/ library/documents/economics_for_coastal_marine_management/gulfcoast-recovery-esa. doc

Wang, Y., Wang, H., Wang, J., Wu, J., \& Liu, X. (2013). Prevalence and predictors of posttraumatic growth in accidentally injured patients. Journal of Clinical Psychology in Medical Settings. doi:10.1007/s10880012-9315-2

Weems, C. F., Pina, A. A., Costa, N. M., Watts, S. E., Taylor, L. K., \& Cannon, M. F. (2007). Pre-disaster trait anxiety and negative affect predict posttraumatic stress in youths after Hurricane Katrina. Journal of Consulting and Clinical Psychology, 75, 154-159. doi:10.1037/0022006X.75.1.154

Weiss, D. S., \& Marmar, C. R. (1997). The Impact of Events ScaleRevised. In J. P. Wilson \& T. M. Kean (Eds.), Assessing psychological trauma and PTSD: A practitioner's handbook (pp. 399-411). New York, NY: Guilford Press.

White, I. K., Philpot, T. S., Wylie, K., \& McGowen, E. (2007). Feeling the pain of my people: Hurricane Katrina, racial inequality, and the psyche 
of Black America. Journal of Black Studies, 37, 523-538. doi:10.1177/ 0021934706296191

Wilson, J. T., \& Boden, J. M. (2008). The effects of personality, social support, and religiosity on posttraumatic growth. Australasian Journal of Disaster and Trauma Studies, 2008, 1-19. Retrieved from http:// www.massey.ac.nz/ trauma/

Xu, J., \& Liao, Q. (2011). Prevalence and predictors of posttraumatic growth among adult survivors one year following 2008 Sichuan earthquake. Journal of Affective Disorders, 133, 274-280. doi:10.1016/j.jad .2011.03.034

Zoellner, T., \& Maercker, A. (2006). Posttraumatic growth in clinical psychology: A critical review and introduction of a two component model. Clinical Psychology Review, 26, 626-653. doi:10.1016/j.cpr .2006.01.008

Zoellner, T., Rabe, S., Karl, A., \& Maercker, A. (2011). Posttraumatic growth as outcome of a cognitive-behavioural therapy trial for motor vehicle accident survivors with PTSD. Psychology and Psychotherapy: Theory, Research, and Practice, 84, 201-213. doi:10.1348/ $147608310 \times 520157$

Received September 6, 2012

Revision received April 11, 2013

Accepted April 29, 2013

\section{Members of Underrepresented Groups: Reviewers for Journal Manuscripts Wanted}

If you are interested in reviewing manuscripts for APA journals, the APA Publications and Communications Board would like to invite your participation. Manuscript reviewers are vital to the publications process. As a reviewer, you would gain valuable experience in publishing. The P\&C Board is particularly interested in encouraging members of underrepresented groups to participate more in this process.

If you are interested in reviewing manuscripts, please write APA Journals at Reviewers@apa.org. Please note the following important points:

- To be selected as a reviewer, you must have published articles in peer-reviewed journals. The experience of publishing provides a reviewer with the basis for preparing a thorough, objective review.

- To be selected, it is critical to be a regular reader of the five to six empirical journals that are most central to the area or journal for which you would like to review. Current knowledge of recently published research provides a reviewer with the knowledge base to evaluate a new submission within the context of existing research.

- To select the appropriate reviewers for each manuscript, the editor needs detailed information. Please include with your letter your vita. In the letter, please identify which APA journal(s) you are interested in, and describe your area of expertise. Be as specific as possible. For example, "social psychology" is not sufficient-you would need to specify "social cognition" or "attitude change" as well.

- Reviewing a manuscript takes time (1-4 hours per manuscript reviewed). If you are selected to review a manuscript, be prepared to invest the necessary time to evaluate the manuscript thoroughly.

APA now has an online video course that provides guidance in reviewing manuscripts. To learn more about the course and to access the video, visit http://www.apa.org/pubs/authors/reviewmanuscript-ce-video.aspx. 